\title{
BMJ Open The effect of an interdisciplinary rehabilitation intervention comparing HRQoL, symptom burden and physical function among patients with primary glioma: an RCT study protocol
}

\author{
Anders Hansen, ${ }^{1}$ Lisbeth Kirstine Rosenbek Minet, ${ }^{2}$ Karen Søgaard, ${ }^{3}$ Jens Ole Jarden ${ }^{4}$
}

To cite: Hansen A, Rosenbek Minet LK, Søgaard K, et al. The effect of an interdisciplinary rehabilitation intervention comparing HRQoL, symptom burden and physical function among patients with primary glioma: an RCT study protocol. BMJ Open 2014;4:e005490. doi:10.1136/bmjopen-2014005490

- Prepublication history for this paper is available online To view these files please visit the journal online (http://dx.doi.org/10.1136/ bmjopen-2014-005490).

Received 16 April 2014 Revised 29 August 2014 Accepted 11 September 2014

\section{CrossMark}

${ }^{1}$ Rehabilitation Unit, Odense University Hospital, Odense, Fyn, Denmark

${ }^{2}$ Institute of Clinical Research, University of Southern Denmark, Odense, Denmark ${ }^{3}$ Institute of Sports Science and Clinical Biomechanics, University of Southern Denmark, Odense, Denmark ${ }^{4}$ Department of Neurology, Herlev University Hospital, University of Copenhagen, Copenhagen, Denmark

Correspondence to Anders Hansen; anders.hansen@rsyd.dk, http://www.ouh.dk

\section{ABSTRACT}

Introduction: Gliomas are among the biggest challenges in neurological and oncology rehabilitation and optimising treatment is of major clinical importance in this population. Although inpatient rehabilitation among glioma patients' results in improved functional measures, rehabilitation efforts are still not emphasised in this patient group and the literature lacks studies investigating the impact of outpatient rehabilitation.

Method: This protocol describes a randomised 6-week parallel group rehabilitation study investigating an outpatient interdisciplinary rehabilitation programme. The intervention consists of 6 weeks intensive physiotherapy as groups exercise in conjunction with 0-6 weeks of individual occupational therapy if a need is present. The aim of this study is to describe the design of the upcoming randomised control trial (RCT). The results of the RCT will add to the growing body of literature investigating the potential role of exercise as a supportive therapeutic intervention for a patient with cancer.

Ethics and dissemination: The project is approved by the Regional Scientific Ethical Committees for Southern Denmark under Project-ID: (S-20140108) and by the Danish Data Protection Agency (J. no.2008-58-0035). Dissemination will occur through presentation and findings will be published in peer-reviewed journals. The key strength of this study is its randomised design and it is the first study to investigate a standardised outpatient interdisciplinary rehabilitation programme among patients with glioma. A potential limitation is the uncertainty and risk of side effects to the concomitant treatment, which enhances the risk of dropout.

Trial registration number: ClinicalTrials.gov Identifier NCT02221986.

\section{BACKGROUND}

Primary brain tumour is a complicated condition due to complex diagnostic and treatment regimes. It has a progressive nature and a poor prognosis causing $2 \%$ of all cancer- related deaths. ${ }^{12}$ Gliomas are the most frequent primary neoplasm in the central nervous system ${ }^{3}$ and, according to $\mathrm{WHO},{ }^{4}$ histologically categorised into low-grade glioma (LGG) (WHO grades I/II) or high-grade glioma (HGG) (WHO grades III/IV). Gliomas are among the biggest challenges in neurological and oncology rehabilitation ${ }^{5} 6$ and optimising treatment is of major clinical importance in this population. Patients often have neurological deficits such as sensory and motor, cognitive, functional deficits (hemiparesis, dysphasia, ataxia) and psychosocial (personality changes, loss of insight or empathy) factors due to tumour localisation or remote effects. ${ }^{7} 8$ Not only does the damage to the brain tissue from tumour result in neurological impairment, the treatments can produce significant side effects including severe muscle weakness, fatigue, headache, vomiting and insomnia. ${ }^{9} 10$ The majority of patients have multiple impairments often resulting in great suffering and low health-related quality of life (HRQoL) ${ }^{79-}$ ${ }^{11}$ Since the vast majority of patients cannot be cured, outcome measures in clinical cancer research have traditionally focused on prolonging the overall survival, progressionfree survival or response to the medical treatment. ${ }^{12-15}$ Today there is a general agreement that HRQoL measures are increasingly important and The American Society of Clinical Oncology has suggested that QoL measurements are an important primary end point in any phase III study. ${ }^{16}$ HRQoL plays a role in predicting survival or survival duration ${ }^{17}$ among standard prognostic indicators such as histology and clinical stage. However, research on glioma patients' perception on HRQoL is sparse compared with other patient categories with neoplasms. ${ }^{13} 18$ 
Advances in neurosurgical techniques and medical treatment have resulted in an increased survival time. ${ }^{19-23}$ This has led to a pronounced proportion of patients having a rehabilitation need. ${ }^{24}$ Several studies indicate that patients with glioma receiving inpatient rehabilitation acquire significant HRQoL, cognitive and functional gains similar to those seen in patients with non-neoplastic neurological

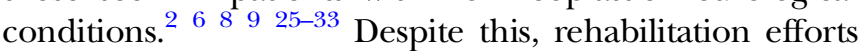
are still not emphasised in this patient group 2429303435 and a Cochrane review recently concluded that no welldesigned clinical trials have investigated the effect of multidisciplinary rehabilitation in patients with brain tumour. Available data are often limited by small sample size studies, ${ }^{27}{ }^{30}$ heterogeneous diagnostic groups with histologically mixed tumour types, ${ }^{29}{ }^{32}$ and missing details concerning resection and characteristics of the tumour, including size and location or information regarding neurological deficits or treatment to date. ${ }^{2}{ }^{32}$ In the present study, interdisciplinary rehabilitation is defined as the coordinated distribution of interdimensional rehabilitation (such as physiotherapy, occupational therapy, nursing, psychology and other allied health interventions) to improve symptoms, maximising functional independence and participation by using a holistic biopsychosocial model (covering physical and psychosocial aspects) of care, as defined by The International Classification of Functioning, Disability and Health (ICF). ${ }^{36}$

Little is known of the functional path of patients with glioma in the outpatient rehabilitation phase and it has been suggested that prospective studies should test the effect of properly selected training interventions to avert and/or relieve dysfunction. ${ }^{53}$ To the best of our knowledge, no randomised studies have investigated whether a standardised outpatient interdisciplinary rehabilitation programme among patients with glioma has effect on HRQoL, symptom burden or physical function. The results of the present RCT study will add to the growing body of literature investigating the potential role of exercise as a supportive therapeutic intervention for patients with cancer.

We hypothesise that patients attending an interdisciplinary rehabilitation programme of intensive specialised physiotherapy and occupational therapy will improve their perception of HRQoL, reduce symptom burden and maintain or delay regression in physical function (defined as muscle strength, $\mathrm{VO}_{2 \text { peak }}$, balance, gait function and activity levels).

\section{OBJECTIVES}

The primary objective is to investigate if a structured rehabilitation programme of intensive specialised physiotherapy and occupational therapy versus standard care has effect on HRQoL. The secondary objective is to investigate whether the rehabilitation programme can reduce the symptom burden and maintain or delay regression in physical function.

\section{TRIAL DESIGN}

This trial is designed as a randomised, controlled, outcome assessor blinded, interdisciplinary exploratory trial with parallel groups.

\section{STUDY SETTING}

The intervention is set to begin in November 2014 at Odense University Hospital (OUH) in the Region of Southern Denmark and end in early spring 2017. With a regional population of 1.2 million, approximately 90 patients are annually diagnosed with glioma (WHO grades I-IV) at OUH.

\section{ELIGIBILITY CRITERIA}

Patient eligibility for randomisation and inclusion must comply with: (1) diagnosis of primary glioma (WHO grades I-IV), (2) age $\geq 18$, reference with diagnosis or treatment at Odense University Hospital, (3) Karnofsky performance score (KPS) $\geq 70$ and (4) ability to understand Danish. Exclusion criteria are (1) pregnancy, (2) known psychiatric diagnosis or substance abuse, (3) heart problems excluding intense exercise (New York Heart Association (NYHA) group III and IV) and (4) pronounced impressive or expressive aphasia. The reason for excluding KPS $<70$ is to ensure inclusion of patients able to conduct the physical training at an active and independent level, having cognitive ability to complete questionnaires and socially be able to interact with others.

\section{INTERVENTION}

The intervention consists of 6 weeks of intensive outpatient physiotherapy in conjunction with $0-6$ weeks of occupational therapy if a need is indicated. The physical intervention contains supervised group exercise of 90 min three times a week in groups of up to four patients included continuously. Exercise includes individually tailored strength training of main muscle groups with increasing load ranging from 15 to 10 repetition maximum (RM) (leg press, arm flexion, arm extension, knee flexion and knee extension), cardiotraining (20 min of cycling or treadmill with intensities ranging from $65 \%$ to $85 \%$ of the heart rate reserve), body awareness training or relaxation (training of proprioception, postural control or stability of the core muscles tailored to personal needs). Every session starts with 5-10 min of warm up. The strength training workload is calculated based on baseline tests and patients follow a training diary with instructions to progression. The cardiovascular training is monitored by pulse through means of a wireless heart rate transmitter worn by the patients.

The occupational therapy intervention consists of individual training $60 \mathrm{~min}$ twice a week for patients having deficits in activity or participation levels measured by the Assessment of Motor and Process Skills (AMPS). The 
training focuses on bettering the patients' functional capacity, body, activity and participation level by adapting activities, regaining or developing activity abilities and/ or rebuilding and developing patient skills. ${ }^{37}$ The occupational therapy is terminated before the sixth week if the patient has reached the appointed functional goals and follow-up assessments are conducted.

The control group receives usual standard of care (eg, no training, individual training or group training in the municipality). The amount of training in this group is based on a questionnaire at the follow-up trials.

\section{SAFETY}

Prior to each physical training session the study nurse assesses each individual patient for the following conditions: diastolic blood pressure $<45$ or $>100$, pulse $>100$, temperature above $38^{\circ} \mathrm{C}$, respiration frequency at rest $>20$, infection requiring treatment with antibiotics, ongoing bleeding; fresh petechiae, bruises, ${ }^{38}$ blood leucocytes $<5 \times 10^{9} / \mathrm{L}$ and blood thrombocytes $<5 \times 10^{3} / \mu \mathrm{L}$. If a condition is found, the patient will be excluded from the physical workout on that specific day and a physician will be informed. All patients are instructed to interrupt or stop training at any time if they feel faint or unwell.

\section{FEASIBILITY STUDY}

Before initiating the RCT-study a feasibility study on 24 patients was conducted to (1) test the feasibility and safety of a 6-week interdisciplinary rehabilitation

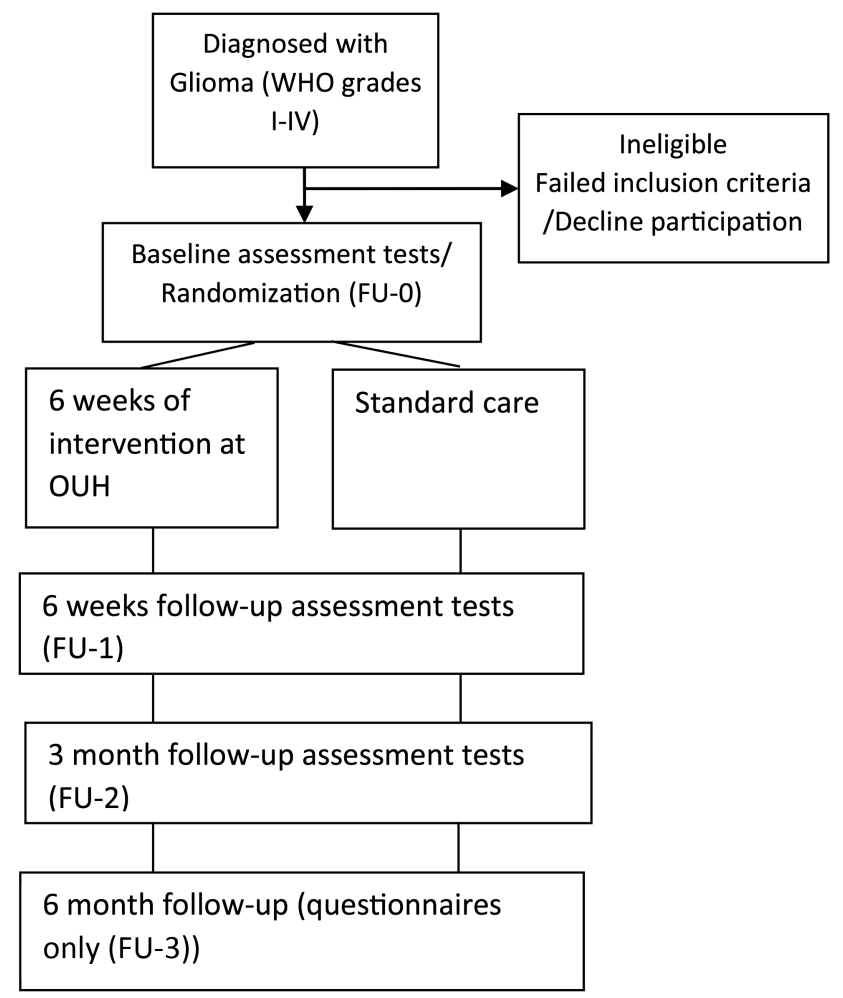

Figure 1 Overview of the study design. programme of individually activity-based training and physical exercise as a group intervention for patients with primary glioma, (2) to assess the preliminary effects on HRQoL, symptom burden and physical function and (3) to determine the effect sizes and sample needed for the RCT-study. The feasibility study also informed the intensity and progression in the final programme and secured that the training was well tolerated by this specific group of patients.

\section{CRITERIA FOR DISCONTINUING ALLOCATED INTERVENTION FOR A GIVEN TRIAL PARTICIPANT}

It is to be expected that some patients will experience side effects to their concomitant treatment with radiation, chemotherapy or cortisone. For a given patient, the assigned study intervention will be discontinued at the discretion of the trial investigators if the healthcare staff members notice a change in behaviour occurring in the patient or the symptom burden is too high. The physical and emotional status of the patients are evaluated before every training session. If patients exceed the set of specified requirements, they are referred to the study nurse and neurologist for further evaluation and possible exclusion. Regardless of any decision to modify or discontinue the assigned intervention, the patient is retained in the trial whenever possible to enable follow-up data collection and to prevent missing data. Patients are informed that they can retrieve their consent at any time without any consequences regarding their relationship with the staff or the content of their medical treatment. Patients are referred to municipality rehabilitation if the specialised treatment does not meet the patients' expectations.

\section{USEFULNESS OF THE TRIAL}

Patients allocated to the intervention group will likely exercise more than usual. However, experience shows that many associate this with increased profits. ${ }^{39}$ This study is carried out so that we can, in the future, organise the best possible rehabilitation for patients with glioma.

\section{SIDE EFFECTS, RISKS, COMPLICATIONS AND DRAWBACKS}

We do not anticipate side effects associated with the rehabilitation. On the contrary, we expect patients to experience similar positive effects on the treatment-related side effects as other cancer groups. ${ }^{39-41}$

\section{OUTCOME}

All outcome measures are gathered at baseline (FU-0) at the end of the sixth week of intervention (FU-1), at a 3-month follow-up (FU-2) and at a 6-month follow-up (questionnaires only; FU-3; see figure 1). At baseline, descriptive variables for each participant in terms of gender, age, weight, height and cohabiting is assessed. Disease variables and treatments are obtained through review of medical records. Extent of resection is assessed 
through imaging definitions $72 \mathrm{~h}$ postoperative. Data on tumour structures, residual tumour, infarct or haematoma in or around the cavity and tumour localisation are extracted.

All physical assessment tools and questionnaires are set to be conducted within $90 \mathrm{~min}$ and occupational tests within $60 \mathrm{~min}$. These assessments are done over a 2-day span to decrease the participant burden and avoid risk of bias due to fatigue.

The tests battery includes two questionnaires: The European Organization for Research and Treatment of Cancer Quality of Life Questionnaire C30 (EORTC-QLQ-C30) and the EORTC brain cancer module (EORTC QLQ-BN20) on patients' perception on HRQoL and symptom burden. Five physiological tests measure physical function defined as: estimated maximum muscle strength (knee extension, knee flexion, arm flexion, arm extension and leg press), maximal oxygen uptake $\left(\mathrm{VO}_{2 \text { peak }}\right)$, balance, gait velocity and steps frequency and activity levels (see table 1).

\section{SAMPLE SIZE}

According to the scoring manual for EORTC QLQ-30 a change of 10 points or more is considered to be a moderate to large clinically significant change. Based on this assumption and results of the feasibility study $(n=24)$, a sample size is calculated. At an expected 'effect size' of at least 10 points $(\mathrm{SD} \pm 24,6)$ increase in the EORTC QLQ-30 General Health Scale/QoL (paragraphs 29 and 30 ) with a statistical power of $\beta 0.8$ and $\alpha$ of 0.05 the study requires 48 participants in each arm. To meet an expected dropout rate of approximately $15 \%$ a total of 56 participants will be included in each group. Based on 90 new cases annually and acceptance and fulfilment of inclusion criteria based on the feasibility study of $>80 \%$, approximately 64 patients will be included per year.

\section{RECRUITMENT}

On a daily basis the administration list from neurological and neurosurgical departments is screened for potential participants by the study nurse. Concurrently, a nurse from the neurosurgical department supplies information on planned cerebral tumour operations. The study leader/nurse approaches eligible patients at the neurosurgical department within $24 \mathrm{~h}$ after returning from the intensive recovery room when the first contact with the therapist is normally scheduled. A KPS is estimated and permission to pass information on to a neurology specialist for histological assessment of inclusion/exclusion criteria is obtained. Before discharge (typically at the fourth postoperative day) the patient is approached a second time for oral information with the opportunity for relatives to be present. After $24 \mathrm{~h}$ (or the nearest weekday) postdischarge, the study leader/nurse contacts the patient by telephone and gets accept or refusal for study participation. If accepted, the patient will receive a formal invitation for baseline assessing through the mail. For HGG this is simultaneously with the start of the radiation treatment approximately 4 weeks postdischarge. For patients with LGG not offered radiation treatment, the start of intervention is likewise 4 weeks postdischarge. Informed consent is obtained at the baseline test.

\section{RANDOMISATION}

Participants are randomly assigned to a control or intervention group with a 1:1 allocation by block randomisation stratified by LGG versus HGG. The block size will not be disclosed to sustain concealment. Consecutively, closed, opaque, numbered envelopes containing assembly allocation are prepared by an assistant outside the study group. The envelopes are stored securely in a locked container. After baseline assessment tests the randomisation is performed by a secretary with no interest

\begin{tabular}{|c|c|c|}
\hline Variable & Details & Unit \\
\hline \multicolumn{3}{|l|}{ Primary outcome } \\
\hline HRQoL & EORTC-QLQ-C30 & Score \\
\hline \multicolumn{3}{|l|}{ Secondary outcomes } \\
\hline Symptom burden & EORTC-QLQ-30 with the addition of BN-20 & Score \\
\hline Estimated maximum muscle strength of leg press & $3-8 \mathrm{RM}$ & $\mathrm{kg}$ \\
\hline Estimated maximum muscle strength of knee extension & 3-8 RM & $\mathrm{kg}$ \\
\hline Estimated maximum muscle strength of knee flexion & 3-8 RM & $\mathrm{kg}$ \\
\hline Estimated maximum muscle strength of arm flexion & 3-8 RM & $\mathrm{kg}$ \\
\hline Estimated maximum muscle strength of arm extension & 3-8 RM & $\mathrm{kg}$ \\
\hline Peak oxygen uptake $\left(\mathrm{VO}_{2 \text { peak }}\right)$ & Åstrand-Rhyming cycle test & $\mathrm{mL} . \mathrm{O}_{2} / \mathrm{min} / \mathrm{kg}$ \\
\hline Standing balance & Sway test & $95 \% \mathrm{Cl}$ \\
\hline Gait velocity & 10 (10MWT) & sek. \\
\hline Number of steps & 10(10MWT) & Steps \\
\hline Activity levels & The AMPS & Score \\
\hline Physical activity levels & Questionnaire & Score \\
\hline
\end{tabular}


in the outcome of the study. The nurse will open the envelope and reveal the allocation for the patient.

\section{BLINDING}

Owing to the nature of the intervention neither participants nor staff can be blinded to allocation but are instructed not to reveal the allocation status of the participant at the follow-up assessments. The testing personnel are blinded to which intervention the patients have received. An employee outside the research team will feed data into the computer in separate datasheets so that the researchers can analyse data without having access to information about the allocation.

\section{DATA COLLECTION METHODS}

HRQoL is assessed by means of the questionnaire EORTC-QLQ- $30^{43}$ with the addition of the questionnaire EORTC-BN20. These are handed out at baseline tests to be completed at the hospital.

The EORTC QLQ-C30 ${ }^{43}$ consists of single-item and multi-item scales. There are 30 items of which 24 cumulate into 9 multi-item scales representing various HRQoL dimensions: 5 functioning scales (physical, role, emotional, cognitive and social), 3 symptom scales (fatigue, pain, and nausea and vomiting) and a global health status/QoL. Six single item scales assess treatment-related symptoms: dyspnoea, loss of appetite, sleep disturbance, constipation, diarrhoea and perceived financial consequences of the treatment. EORTC-BN20 demonstrates sufficient psychometric properties and is used in conjunction with the EORTC QLQ-C30 for assessing the HRQoL of patients with brain tumour. ${ }^{13}$ The EORTC-BN20 questionnaire contains 20 items of which 13 cumulate into 4 multi-item scales representing: future uncertainty, visual disorder, motor dysfunction, communication deficit and 7 single items (headaches, seizures, drowsiness, hair loss, itchy skin, weakness of legs and bladder control). ${ }^{13}$ All raw scores of the EORCT QLQ-C30 and EORTC-BN20 are linearly changed to a $0-100$ scale. High scores indicates a better perceived HRQoL for the global health status/QoL and functioning scales and worse score for the treatment-related symptom scale.

\section{PHYSICAL FUNCTION}

Muscle strength is assessed by indirect $1 \mathrm{RM}$ tests. The tests involve performance on TuffStuff variable resistance equipment and target large muscle groups as follows: (1) leg press (knee extensors, hip extensors, hip adductors and ankle joint flexors), (2) arm flexion (musculus biceps brachii, musculus brachialis and musculus brachioradialis), (3) arm extension (musculus triceps brachii), (4) knee extension (musculus quadriceps femoris) and (5) knee flexion (musculus satorius, musculus gracilis, musculus biceps femoris, musculus semimembranosus, musculus semitendinosus, musculus gastrocnemius and musculus plantaris).
The patients follow a familiarisation protocol of performing a set of 12 submaximal repetitions with a load equivalent to an estimated $50 \%$ (educated guess) of a 1 RM followed by a 2-min break. The physiotherapist focuses on correction technique, breathing technique and execution of the habituation set. The patient then performs another habituation set of eight-submaximal repetitions with a load equal to $75 \%$ (educated guess) of 1 RM. After a 2-min break the actual RM test is started. The test is performed by the physiotherapist adding loads until the patient is expected to reach failure within 3-8 repetitions or the patient voluntarily stops. If the load can be carried nine times or more, the test is discontinued, and a break of $2 \mathrm{~min}$ is given before a heavier load is attempted. A load equivalent of 3RM-8RM has to be located within one to four trials (exclusive of the habituation sets). Otherwise, the test is dismissed due to fatigue. The tester strictly observes each repetition and only trials completed with proper form through the full range of motion are counted. Participants are encouraged to complete repetitions consecutively and verbal motivation is standardised using a protocol during all testing sessions. An equation proposed by Brzycki ${ }^{45}$ is used to estimate $1 \mathrm{RM}$.

Measuring $\mathrm{VO}_{2 \text { peak }}$ directly is considered the gold standard but requires refined equipment, skilled technicians, time and money. It is also, for a number of participants, associated with discomfort ${ }^{46}$ and neurological specific impairments such as muscle weakness, fatigue, poor balance or spasticity and can interfere with the patients' ability to reach maximum function using standard maximal exercise. There are no validated submaximal fitness tests for these patients, but the Asstrand-Rhyming one-point bicycle test is considered the best choice for safety reasons, when a maximum test is associated with risk. The Åstrand-Rhyming test estimates maximal oxygen uptake (fitness indicators) from workload and pulse rate as per protocol by Åstrand. ${ }^{47}$ The participant cycles for $6 \mathrm{~min}$ at 50-60 rpm with a load that stabilises the pulse in the range of $110-170 \mathrm{bpm}$, at the last of the $6 \mathrm{~min}$.

Balance is assessed by a sway test. Laboratory-based assessment using measures of centre of pressure (COP) recorded from a force platform is considered the gold standard measure of balance. ${ }^{48}$ The Wii Balance Board (WBB) is a valid and low-cost system for assessing standing balance. ${ }^{48}$ Good-to-excellent test-retest reproducibility has been demonstrated during a static bilateral stance in 30 young individuals by extracting raw vertical force data from the WBB. ${ }^{48}$ The WBB makes it possible to obtain non-invasive data on participants by four piezoelectric strain gauges built into the corners of the device. The outputs of these gauges assess force distribution and the resultant movements in COP through a Bluetooth connection. ${ }^{49}$ Sway measurements are assessed by a protocol previously used by Jørgensen $e t a l^{50}$

Gait velocity and step frequency is assessed by a $10 \mathrm{~m}$ walk test (10MWT) as per protocol of Watson, ${ }^{51}$ a valid 
and reliable quantitative test to measure walking ability in patients with neurological disorders. The patient walks $10 \mathrm{~m}$ from a standing position at a preferred speed. Patients are allowed to use a preferred aid if needed. Time is recorded using a stopwatch and the number of steps taken is counted. Mean time score and mean number of steps is calculated.

Changes in activity and performance status are assessed by an AMPS test, which is a globally recognised assessment of everyday function. The AMPS is an observational, standardised performance-based assessment that obtains information on the quality of an individual's performance of activity of daily living (ADL) tasks. ${ }^{52}$ According to AMPS, a higher score indicates an increased level of independence, increased safety in the community and increased efficiency of performance. ${ }^{52}$

Physical activity levels at work and in leisure time are assessed through questions inspired by Saltin and Grimby. ${ }^{42}$

\section{RETENTION}

Once a patient is randomised the study staff will make every effort to follow the patient for the entire study period. The staff is accountable for developing and implementing standard operating procedures to maximise level of follow-up, and for limiting participant burden related to visits and procedures. Before each follow-up the patient will receive a telephone call from the study leader or nurse and receive a formal invitation through the mail. The nurse will give the study group an update on the patient's health status. If a patient has experienced a heavy disease progression or is not physically or mentally able to participate, she/he will be lost to follow-up in order to protect her/his safety. Participants may withdraw from the study for any reason at any time without it affecting the primary treatment.

\section{STATISTICAL METHOD}

The intervention group will be compared with controls for all primary analysis. We will use $\chi$ tests for binary outcomes and t tests or Mann-Whitney for continuous outcomes. For subgroup analyses, we will use regression methods with appropriate interaction terms (respective subgroup $\times$ treatment group). Multivariable analyses will be based on logistic regression for binary outcomes and linear regression for continuous outcomes. All analyses are conducted using SPSS V.21 for Windows. For all tests two-sided $p$ values with $\alpha=<0.05$ level of significance are used. A Bonferroni method is used to appropriately adjust the overall level of significance for multiple primary and secondary outcomes.

\section{MISSING DATA}

Linear Mixed Models and Generalised Estimating Equations are used for handling non-ignorable dropouts in the longitudinal study.

\section{CONFIDENTIALITY}

All study-related information will be stored securely at the study site in accordance with the Danish Data Protection Agency. All participant information will be stored in locked file cabinets in areas with limited access. All reports, data collection, process and administrative forms will be identified by a coded ID number to maintain participant confidentiality. All records containing names or other personal identifiers, such as informed consent forms, are stored separately from study records and identified by code number. All local databases will be secured with password-protected access systems.

Contributors AH, LKRM, KS and JOJ were involved in the contribution and design of the study. All authors have read and approved the final manuscript.

Competing interests None.

Patient consent Obtained.

Ethics approval The project was approved by the Regional Scientific Ethical Committees for Southern Denmark under Project-ID: (S-20140108) and by the Danish Data Protection Agency (J. no.2008-58-0035).

Provenance and peer review Not commissioned; externally peer reviewed.

Open Access This is an Open Access article distributed in accordance with the Creative Commons Attribution Non Commercial (CC BY-NC 4.0) license, which permits others to distribute, remix, adapt, build upon this work noncommercially, and license their derivative works on different terms, provided the original work is properly cited and the use is non-commercial. See: http:// creativecommons.org/licenses/by-nc/4.0/

\section{REFERENCES}

1. Khan F, Amatya B. Factors associated with long-term functional outcomes, psychological sequelae and quality of life in persons after primary brain tumour. J Neurooncol 2013;111:355-66.

2. Bartolo M, Zucchella C, Pace A, et al. Improving neuro-oncological patients care: basic and practical concepts for nurse specialist in neuro-rehabilitation. J Exp Clin Cancer Res 2012;31:82.

3. Stupp R, Gander M, Leyvraz S, et al. Current and future developments in the use of temozolomide for the treatment of brain tumours. Lancet Oncol 2001;2:552-60.

4. Louis DN, Ohgaki H, Wiestler OD, et al. The $2007 \mathrm{WHO}$ classification of tumours of the central nervous system. Acta Neuropathol 2007;114:97-109.

5. Jones LW, Friedman AH, West MJ, et al. Quantitative assessment of cardiorespiratory fitness, skeletal muscle function, and body composition in adults with primary malignant glioma. Cancer 2010;116:695-704.

6. Vargo M. Brain tumor rehabilitation. American journal of physical medicine \& rehabilitation/Association of Academic Physiatrists 2011;90(5 Suppl 1):S50-62.

7. Mukand JA, Blackinton DD, Crincoli MG, et al. Incidence of neurologic deficits and rehabilitation of patients with brain tumors. Am J Phys Med Rehabil 2001;80:346-50.

8. Huang ME, Cifu DX, Keyser-Marcus L. Functional outcome after brain tumor and acute stroke: a comparative analysis. Arch Phys Med Rehabil 1998;79:1386-90.

9. Khan F, Amatya B, Ng L, et al. Multidisciplinary rehabilitation after primary brain tumour treatment. Cochrane Database Syst Rev 2013;1:CD009509.

10. Jones LW, Mourtzakis M, Peters KB, et al. Changes in functional performance measures in adults undergoing chemoradiation for primary malignant glioma: a feasibility study. Oncologist 2010;15:636-47.

11. Jones LW, Guill B, Keir ST, et al. Patterns of exercise across the cancer trajectory in brain tumor patients. Cancer 2006;106:2224-32.

12. van den Bent MJ, Carpentier AF, Brandes AA, et al. Adjuvant procarbazine, lomustine, and vincristine improves progression-free survival but not overall survival in newly diagnosed anaplastic oligodendrogliomas and oligoastrocytomas: a randomized European 
Organisation for Research and Treatment of Cancer phase III trial. $J$ Clin Oncol 2006;24:2715-22.

13. Taphoorn MJ, Claassens L, Aaronson NK, et al. An international validation study of the EORTC brain cancer module (EORTC QLQ-BN20) for assessing health-related quality of life and symptoms in brain cancer patients. Eur J Cancer 2010;46:1033-40.

14. Shields LB, Choucair A, Choucair AK. Quality of life measures as a preliminary clinical indicator in patients with primary brain tumors. Surg Neurol Int 2013;4:48.

15. Bosma I, Reijneveld JC, Douw L, et al. Health-related quality of life of long-term high-grade glioma survivors. Neuro Oncol 2009;11:51-8.

16. [No authors listed]. Outcomes of cancer treatment for technology assessment and cancer treatment guidelines. American Society of Clinical Oncology. J Clin Oncol 1996;14:671-9.

17. Mauer ME, Bottomley A, Taphoorn MJ. Evaluating health-related quality of life and symptom burden in brain tumour patients: instruments for use in experimental trials and clinical practice. Curr Opin Neurol 2008;21:745-53.

18. Cheng JX, Zhang X, Liu BL. Health-related quality of life in patients with high-grade glioma. Neuro Oncol 2009;11:41-50.

19. McGirt MJ, Chaichana KL, Gathinji M, et al. Independent association of extent of resection with survival in patients with malignant brain astrocytoma. J Neurosurg 2009;110:156-62.

20. Stummer W, Reulen HJ, Meinel T, et al. Extent of resection and survival in glioblastoma multiforme: identification of and adjustment for bias. Neurosurgery 2008;62:564-76; discussion 64-76.

21. Wen PY, Schiff D, Kesari S, et al. Medical management of patients with brain tumors. J Neurooncol 2006;80:313-32.

22. Stupp R, Hegi ME, Mason WP, et al. Effects of radiotherapy with concomitant and adjuvant temozolomide versus radiotherapy alone on survival in glioblastoma in a randomised phase III study: 5 -year analysis of the EORTC-NCIC trial. Lancet Oncol 2009;10:459-66.

23. Stupp R, Mason WP, van den Bent MJ, et al. Radiotherapy plus concomitant and adjuvant temozolomide for glioblastoma. $N$ Engl J Med 2005;352:987-96.

24. Geler-Kulcu D, Gulsen G, Buyukbaba E, et al. Functional recovery of patients with brain tumor or acute stroke after rehabilitation: a comparative study. J Clin Neurosci 2009;16:74-8.

25. Roberts PS, Nuno M, Sherman D, et al. The impact of inpatient rehabilitation on function and survival of newly diagnosed patients with glioblastoma. PM R 2014;6:514-21.

26. Greenberg E, Treger I, Ring H. Rehabilitation outcomes in patients with brain tumors and acute stroke: comparative study of inpatient rehabilitation. Am J Phys Med Rehabil 2006;85:568-73.

27. Huang ME, Wartella JE, Kreutzer JS. Functional outcomes and quality of life in patients with brain tumors: a preliminary report. Arch Phys Med Rehabil 2001;82:1540-6.

28. Kirshblum S, O'Dell MW, Ho C, et al. Rehabilitation of persons with central nervous system tumors. Cancer 2001;92(4 Suppl):1029-38.

29. Formica V, Del Monte G, Giacchetti I, et al. Rehabilitation in neuro-oncology: a meta-analysis of published data and a mono-institutional experience. Integr Cancer Ther 2011;10:119-26.

30. Tang V, Rathbone M, Park Dorsay J, et al. Rehabilitation in primary and metastatic brain tumours: impact of functional outcomes on survival. J Neurol 2008;255:820-7.

31. Marciniak CM, Sliwa JA, Heinemann AW, et al. Functional outcomes of persons with brain tumors after inpatient rehabilitation. Arch Phys Med Rehabil 2001;82:457-63.
32. O'Dell MW, Barr K, Spanier D, et al. Functional outcome of inpatient rehabilitation in persons with brain tumors. Arch Phys Med Rehabil 1998;79:1530-4.

33. Piil K, Juhler M, Jakobsen J, et al. Controlled rehabilitative and supportive care intervention trials in patients with high-grade gliomas and their caregivers: a systematic review. BMJ Support Palliat Care 2014. doi:10.1136/bmjspcare-2013-000593

34. Pace A, Parisi C, Di Lelio M, et al. Home rehabilitation for brain tumor patients. J Exp Clin Cancer Res 2007;26:297-300.

35. Sherer M, Meyers CA, Bergloff P. Efficacy of postacute brain injury rehabilitation for patients with primary malignant brain tumors. Cancer 1997;80:250-7.

36. World Health Organization. ICF: international classification of functioning, disability and health/World Health Organization. Geneva: World Health Organization, 2001.

37. Fisher AG. Occupational therapy intervention process model: a model for planning and implementing top-down, client-centered, and occupation-based interventions: Three Star Press Incorporated, 2009.

38. Adamsen L, Quist M, Andersen C, et al. Effect of a multimodal high intensity exercise intervention in cancer patients undergoing chemotherapy: randomised controlled trial. BMJ 2009;339:b3410.

39. Adamsen L, Quist M, Midtgaard J, et al. The effect of a multidimensional exercise intervention on physical capacity well-being and quality of life in cancer patients undergoing chemotherapy. Support Care Cancer 2006;14:116-27.

40. Dimeo FC, Stieglitz RD, Novelli-Fischer U, et al. Effects of physical activity on the fatigue and psychologic status of cancer patients during chemotherapy. Cancer 1999;85:2273-7.

41. Dimeo FC. Effects of exercise on cancer-related fatigue. Cancer 2001;92(6 Suppl):1689-93

42. Saltin B, Grimby G. Physiological analysis of middle-aged and old former athletes. Comparison with still active athletes of the same ages. Circulation 1968:38:1104-15.

43. Aaronson NK, Ahmedzai S, Bergman B, et al. The European Organization for Research and Treatment of Cancer QLQ-C30: a quality-of-life instrument for use in international clinical trials in oncology. J Natl Cancer Inst 1993;85:365-76.

44. Maringwa J, Quinten C, King M, et al. Minimal clinically meaningful differences for the EORTC QLQ-C30 and EORTC QLQ-BN20 scales in brain cancer patients. Ann Oncol 2011;22:2107-12.

45. Brzycki M. Strength testing: predicting a one-rep max from repetitions to fatigue. J Phy Educ, Recreation and Dance 1993;64:88-90.

46. Legge BJ, Banister EW. The Astrand-Ryhming nomogram revisited. J Appl Physiol (1985) 1986:61:1203-9.

47. Rodahl Å. Textbook of work physiology. 3rd edn. McGraw Hill, 1986. Side 363-384.

48. Clark RA, Bryant AL, Pua $Y$, et al. Validity and reliability of the Nintendo Wii Balance Board for assessment of standing balance. Gait Posture 2010;31:307-10.

49. Koslucher F, Wade MG, Nelson B, et al. Nintendo Wii Balance Board is sensitive to effects of visual tasks on standing sway in healthy elderly adults. Gait Posture 2012;36:605-8.

50. Jørgensen MB, Ektor-Andersen J, Sjogaard G, et al. A randomised controlled trial among cleaners-effects on strength, balance and kinesiophobia. BMC public health 2011:11:776.

51. Watson MJ. Refining the ten-metre walking test for use with neurologically impaired people. Physiotherapy 2002;88:386-97.

52. Fisher AG, Bray Jones K. Vol. Assessment of motor and process skills.1:development, standardization, and administration manual. 6th edn. Fort Collins, CO: Three Star Press, 2007. 